\title{
Variation for Antioxidant Activity and Antioxidants in Tomato
}

\author{
Peter M. Hanson, Ray-yu Yang, Jane Wu, Jen-tzu Chen, Dolores Ledesma, and \\ Samson C.S. Tsou \\ AVRDC-The World Vegetable Center, Box 42 Shanhua, Tainan 741, Taiwan \\ Tung-Ching Lee \\ Department of Food Science and the Centerfor Advanced Food Technology, Rutgers, the State University \\ of New Jersey, 65 Dudley Road, New Brunswick, NJ 08901-8520
}

\begin{abstract}
AdDITIONAL INDEX WORDs. Lycopersicon sp., nutrition, carotenoids, vitamins
Abstract. Tomato (Lycopersicon esculentum Mill.) is among the most widely consumed vegetables worldwide and an important source of certain antioxidants $\mathrm{AO}$ ) including lycopene, $\beta$-carotene, and vitamin $\mathrm{C}$. Improvement of tomato for content of AO and overall antioxidant activity (AOA) could potentially benefit human health in many countries. We evaluated $50 \mathrm{~L}$. esculentum and three L. pimpinellifolium (L.) Mill. entries for contents of lycopene, $\beta$-carotene, ascorbic acid, total phenolics, and two assays for antioxidant activity [anti-radical power (ARP) and inhibition of lipid peroxidation (ILP)] for 2 years during the same period in south Taiwan. We detected high levels of genetic diversity for the AO and AOA measured in this study. Group means of the L. pimpinellifolium entries were significantly higher than L. esculentum group means for ARP, ILP, lycopene, ascorbic acid, phenolics, and soluble solids concentration, suggesting that introgression of alleles from $L$. pimpinellifolium may have potential to improve cultivated tomato for these traits. Ranking of entries for ILP and ARP were consistent between years, particularly for those entries with the highest means and these assays could be adopted by tomato breeders. Results from ILP and ARP assays were highly correlated $(r=0.82 * *)$ and it would be unnecessary to use both assays for tomato. Lycopene, $\beta$-carotene, ascorbic acid, soluble solids, and total phenolics were all positively correlated with ARP. Among AO, total phenolics content was most closely associated with ARP $(r=0.90 * *)$ and ILP $(r=0.83 * *)$; this suggests that phenolics make a major contribution to AOA in tomato fruit. Fruit size was negatively correlated with ARP $(r=-0.74 * *)$ and $\operatorname{ILP}(r=-0.71 * *)$, indicating that combining large fruit size and high AOA will be challenging.
\end{abstract}

Tomato is one of the most economically important vegetables in the world with over 100 million t produced on $\approx 4$ million ha annually (Food and Agriculture Organization of the United Nations, 2004). In terms of human health, tomato fruit provide significant quantities of $\beta$-carotene, a provitamin A carotenoid, and ascorbic acid. Although densities of ascorbic acid and $\beta$-carotene in tomato are modest compared to some other vegetables, tomato ranks high as a source of vitamins $\mathrm{A}$ and $\mathrm{C}$ in human diets because of high consumption in many countries (Davies and Hobson, 1981; Stevens, 1986). In addition to their importance as a provitamin or vitamin, $\beta$-carotene and ascorbic acid also serve as antioxidants (AO); $\beta$-carotene functions to help prevent and neutralize free radical chain reactions and ascorbic acid is an effective scavenger of superoxide, hydrogen peroxide, singlet oxygen and other free radicals (Clevidence et al., 1997; Omaye and Zhang, 1998). Lycopene, the major carotenoid in tomato fruit, is a powerful $\mathrm{AO}$ and has garnered much attention because of the linkage between lycopene-rich diets and lower risks of certain cancers, heart disease, and age-related diseases (Bramley, 2000; Clinton, 1998; Heber and Lu, 2002; Rao and Agarwal, 1998). Although most phenolic compounds in tomato fruit have disappeared at maturity, fully ripened fruit contain modest quantities of querci-

Received for publication 12 Feb. 2004. Accepted for publication 17 Apr. 2004 Journal paper no. JP 175 and publication no. 04-565 of the AVRDC-The World Vegetable Center. We thank the Taiwan Council of Agriculture (Project No. 90AS-1.9-ID-11 and (91-92)AS-1-4-ID-01) for funding this work. We are also grateful to the C.M. Rick Tomato Genetics Resource Center and the USDA, ARS for sharing seed of their Lycopersicon accessions with us and their support of the tomato breeding community worldwide.
tin-3-O-rutinoside (Bovy et al., 2002; Herrmann, 1976), which like other flavonols is a powerful $\mathrm{AO}$ associated with reduced cancer risk (Weisburger, 1999).

Tomato genotypes vary in the types and concentrations of carotenoids (Leonardi et al., 2000; Stommel and Haynes, 1994; Wann et al., 1985), levels of ascorbic acid, and phenolics (Abushita et al., 1997; Lavelli et al., 2000) and selection for higher concentrations of particular $\mathrm{AO}$ is one strategy to improve tomato and other crops for overall antioxidant activity (AOA) (Kochian and Garvin, 1999; Tucker, 2003) However, AO in plants usually occur in mixtures and overall AOA may be greater or lesser than the sum of individual $\mathrm{AO}$ due to synergies or antagonisms (Arnao et al., 2001; Halliwell et al., 1995; Kochian and Gavin, 1999; Stahl et al., 1998), or bioactivity of unknown AO (Arnao et al., 2001).

Evaluation of AOA is complicated because of the multitude of $\mathrm{AO}$ in vegetable extracts, the multifunctionality of some $\mathrm{AO}$, and interactions among AO in mixtures. Different in vitro methods have been designed to estimate AOA by simulating oxidative reactions that could take place during the initiation and development of human disease (Lavelli et al., 2001) but the relative importance of AO in vivo can change, depending on which reactive oxygen species (ROS) is involved, how and where ROS are generated, and the particular target of damage to be measured (Halliwell et al., 1995); consequently, no standard AOA assay has yet been agreed upon (Frankel and Meyer, 2000). AO can directly scavenge or inhibit generation of ROS in vivo (Halliwell et al. 1995); the free-radical trapping assays such as the total radicaltrapping parameter (TRAP), oxygen radical absorbance capacity (ORAC), anti-radical power (ARP) and others estimate AOA by 
generation of ROS and measurement of their disappearance after introduction of AO (Arnao et al. 2001; Cao et al., 1996; Guo et al., 1997; Vinson et al., 1998). AO can also block ROS from causing lipid peroxidation which can begin a series of reactions leading to atherosclerosis (Frankel and Meyer, 2000) and some AOA methods measure the capacity of AO to prevent oxidation of unsaturated lipids (Halliwell et al., 1995).

Because tomato is consumed daily in many countries, production and consumption of tomato cultivars of higher AOA could potentially contribute to better human health worldwide. The objectives of this study were to assess genetic diversity within $L$. esculentum for four AO (lycopene, $\beta$-carotene, ascorbic acid, and total phenolics), and for AOA using two methods (ARP and ILP), and to estimate the association between AOA and specific AO.

\section{Materials and Methods}

Tomato EnTries. Fifty L. esculentum and three L. pimpinellifolium (L.) Mill. entries were included in the study (Table 1). Forty-one of the L. esculentum entries are accessions from the Lycopersicon collection of AVRDC-the World Vegetable Center but were originally gifts from other collections; entries prefixed with "LA" came from the Charles M. Rick Tomato Genetics Resource Center at the Univ. of California-Davis and entries prefixed with "PI" came from the USDA collection in Geneva, N.Y. CLN2026D and FMTT33, are heat-tolerant cultivars bred at the AVRDC - The World Vegetable Center. Nine L. esculentum entries are homozygous for specific genes affecting fruit carotenoid content: T4065 is homozygous for the high pigment $(h p)$ and crimson genes $(o g c)$, T5019 carries the dark green gene $(d g)$ and crimson gene $(o g c)$ genes, and NCEBR-6 carries $o g$; $h p$ and $d g$ increase total carotenoid content and $o g^{\mathrm{c}}$ increases lycopene content at the expense of $\beta$-carotene (Stevens, 1986; Stommel and Haynes 1994); entries 97L63 and CLN1314G have the Beta gene $(B)$, which elevates $\beta$-carotene content at the expense of lycopene (Stommel and Haynes 1994; Tomes and Quackenbush, 1958); LA2996A contains the Delta ( $\mathrm{Del})$ gene, which synthesizes deltacarotene, a carotenoid not normally found in tomato (Stevens and Rick, 1986); the green flesh ( $g f)$ gene present in LA2999 produces fruit with persistent chlorophyll; LA2997 carries the yellow flesh gene $(r)$ that results in fruit with trace amounts of all carotenoids except phytoene; the sherry $(s h)$ gene in LA2644 induces yellow fruit with an orange tinge (Stevens and Rick, 1986).

Entries were grown 2 years in AVRDC - The World Vegetable Center fields from Oct. 2001-Mar. 2002 (2002 season) and Oct. 2002-Mar. 2003 (2003 season). This 6-month period is the most favorable season for tomato production in the lowlands of Taiwan. Mean maximum and minimum temperatures during this period range from 22 to $30^{\circ} \mathrm{C}$ and 12 to $22^{\circ} \mathrm{C}$. Average monthly rainfall is $<50 \mathrm{~mm}$. Entries were replicated twice and arranged in a randomized complete-block design. Plots included five plants in the 2002 season and 10 plants in the 2003 season. Plants were staked and pruned. Fruit at the full red stage were harvested from each plot once when one or more clusters on most plants within plots had ripe fruit. Fruit were taken the same day to the laboratory for sample preparation.

SAMPle PREPARATION. Each sample consisted of $>600 \mathrm{~g}$ fully ripened fruit harvested from a single plot. Fruit were cut, blended with a homogenizer, and filtered through gauze to remove seeds, skin, and membranes. From each sample, six plastic bags were prepared, each containing 10-20 g of tomato slurry. The bags were sealed and immediately stored at $-70{ }^{\circ} \mathrm{C}$ for subsequent analyses of ARP, ILP, carotenoids, ascorbic acid, and total phenolics. Supernatants obtained after centrifugation at $6000 \mathrm{~g}_{\mathrm{n}}$ for $10 \mathrm{~min}$ were used the same day to measure color, and soluble solids concentration.

Chemicals. ABTS (2,2-azino-bis (3-ethylbenz-thiazoline-6sulfonic acid)), HRP (horseradish peroxidase, type VI-A, 1000 unit/mg solid), linoleic acid, $\beta$-carotene, and lycopene were purchased from Sigma Chemical Co. (St. Louis). Trolox (6hydroxy-2,5,7,8-tetramethyl-chroman-2-carboxylic acid) was purchased from Aldrich Co. (St. Louis); and AAPH (2,2-azobis (2-amidino propane) dihydrochloride) was obtained from Wako Co. (Osaka, Japan). Other reagents used in this study were all analytical reagent grade.

Antioxidant activity. Entries were analyzed for AOA by ARP and ILP methods. Both assays are cheap and not laborious, and therefore could possibly be incorporated into breeding programs. ARP was carried out as described in Arnao et al. (2001) with some modifications. This method measures the capacity of different components to scavenge the ABTS radical cation as compared to the standard antioxidant Trolox ( $0-4 \mathrm{~mm})$ in a dose response curve. Trolox is a vitamin $\mathrm{E}$ analog. The $10 \times$ reaction mixture contained $20 \mathrm{~mm}$ ABTS, $0.41 \mathrm{~mm} \mathrm{H}_{2} \mathrm{O}_{2}$ and 50 units of HRP in 50 mM sodium phosphate buffer $(\mathrm{pH7.5})$ in a total volume of $10 \mathrm{~mL}$ and a stable absorbance at $730 \mathrm{~nm}$ was obtained in 2 min. The mixture was then diluted to $1 \times$ with ethanol. Twenty microliters of antioxidant sample with appropriate dilution in water or methanol was added to the $1 \mathrm{~mL}$ of $1 \times$ reaction medium. The decrease in absorbance, which is proportional to the ABTS quenched, was determined after $5 \mathrm{~min}$ by spectrophotometer. The AOA of a sample for the ARP assay was measured within the linear relationship of concentration vs. optical density decrease, and presented as Trolox equivalent (TE) in $\mu \mathrm{mol} \cdot \mathrm{g}^{-1}$ tomato fruit (fresh weight basis).

The ability of antioxidant samples to inhibit lipid peroxidation at pH 7 was tested using linoleic acid as a substrate and AAPH as a free radical generator. Sample antioxidants were obtained from frozen tomato slurry in methanol $(1: 5, \mathrm{w} / \mathrm{v})$ that had been blended and centrifuged. Peroxidation was started by adding 0.1 $\mathrm{mL}$ of $0.1 \mathrm{M} \mathrm{APPH}$ to the reaction mixtures containing $0.21 \mathrm{~mL}$ de-ionized water, $0.165 \mathrm{~mL}$ tetrahydrofuran in $10 \%$ Tween 20 , $1 \mathrm{~mL}$ of $0.2 \mathrm{M}$ phosphate buffer, and $1 \mathrm{~mL}$ of $2.5 \%$ linoleic acid in ethanol. Twenty-five microliters of sample antioxidant were added and the mixture was incubated at $37^{\circ} \mathrm{C}$ for $3 \mathrm{~h}$. After incubation, $0.1 \mathrm{~mL}$ of the mixture was transferred to another tube containing $4.7 \mathrm{~mL}$ of $75 \%$ ethanol followed by adding $0.1 \mathrm{~mL}$ of $30 \%$ ammonium thiocyanate and $0.1 \mathrm{~mL}$ of $0.02 \mathrm{M} \mathrm{FeCl}_{2}$ in $3.5 \% \mathrm{HCl}$. After $3 \mathrm{~min}$, the absorbance was read by spectrophotometer at $500 \mathrm{~nm}$. The absorbance difference of the reaction without linoleic acid and with linoleic acid was represented as $100 \%$ of inhibition. Trolox (0-4 mM) was used as the antioxidant standard and AOA was expressed as TE in $\mu \mathrm{mol} \cdot \mathrm{g}^{-1}$ tomato fruit (fresh weight basis).

Carotenoid contents. Ten grams of frozen tomato slurry were blended with $100 \mathrm{~mL} 6$ hexane : 4 acetone (v/v), and $0.1 \mathrm{~g}$ $\mathrm{MgCO}_{3}$ in a homogenizer. Acetone was then washed out five times with salt-saturated water. The hexane extract was filtered with a $0.45-\mu \mathrm{m}$ filter. Analyses were performed using high-performance liquid chromatography (HPLC; Waters, Milford, Mass.) equipped with a 717 plus autosampler, 600 controller, 2487 detector (read at $436 \mathrm{~nm}$ ) with a $125 \times 4-\mathrm{mm}$ LiChrospher $100 \mathrm{RP}-18 \mathrm{e}$ column, $5 \mu \mathrm{m}$ (Merck, Darmstadt, Germany) under isocratic conditions at ambient temperatures. The mobile phase was 75 acetoniltrile : 
Table 1. Lycopersicon esculentum and L. pimpinellifolium entries evaluated for antioxidant activity, antioxidants, and fruit qualities ${ }^{\mathrm{z}}$, AVRDC-The World

\begin{tabular}{|c|c|c|c|c|c|c|}
\hline \multirow[b]{2}{*}{$\begin{array}{l}\text { Entry name } \\
(\text { carotenoid gene })^{z}\end{array}$} & \multirow[b]{2}{*}{ Species ${ }^{y}$} & \multirow[b]{2}{*}{ Origin } & \multirow[b]{2}{*}{$\begin{array}{l}\text { Fruit size } \\
\text { category }^{x}\end{array}$} & \multicolumn{2}{|c|}{ Antioxidant activity } & \multirow[b]{2}{*}{ Lycopene } \\
\hline & & & & ILPw & ARP & \\
\hline & & & & \multicolumn{2}{|c|}{$\begin{array}{l}\text { Trolox equivalent } \\
\left(\mu \mathrm{mol} \cdot \mathrm{g}^{-1}\right)\end{array}$} & \\
\hline LA 1582 & $\mathrm{P}$ & Peru & very small & $5.77 \pm 0.07$ & $6.84 \pm 0.93$ & $23.09 \pm 0.67$ \\
\hline LA 1586 & $\mathrm{P}$ & Peru & very small & $4.75 \pm 0.79$ & $6.47 \pm 0.72$ & $19.69 \pm 1.10$ \\
\hline LA 1593 & $\mathrm{P}$ & Peru & very small & $4.96 \pm 0.53$ & $6.40 \pm 0.40$ & $18.04 \pm 0.71$ \\
\hline Pusa Ruby & $\mathrm{E}$ & India & large & $4.21 \pm 0.38$ & $3.60 \pm 0.24$ & $6.91 \pm 0.77$ \\
\hline Nova $70-392 \mathrm{~A}$ & $\mathrm{E}$ & USA & large & $3.69 \pm 0.33$ & $3.92 \pm 0.39$ & $7.37 \pm 0.64$ \\
\hline LA 1229 & $\mathrm{E}$ & Ecuador & medium & $3.80 \pm 0.25$ & $3.46 \pm 0.44$ & $5.33 \pm 0.62$ \\
\hline LA 1230 & $\mathrm{E}$ & Ecuador & very large & $3.11 \pm 0.40$ & $3.00 \pm 0.43$ & $5.16 \pm 0.56$ \\
\hline LA1231 & $\mathrm{E}$ & Ecuador & medium & $4.36 \pm 0.68$ & $4.43 \pm 0.35$ & $6.79 \pm 0.50$ \\
\hline LA1247 & $\mathrm{E}$ & Ecuador & very small & $4.49 \pm 0.54$ & $4.59 \pm 0.40$ & $8.93 \pm 0.74$ \\
\hline LA 1289 & $\mathrm{E}$ & Peru & small & $4.80 \pm 0.29$ & $4.88 \pm 0.45$ & $7.22 \pm 1.18$ \\
\hline LA 1308 & $\mathrm{E}$ & Peru & small & $4.83 \pm 0.54$ & $5.74 \pm 0.46$ & $8.17 \pm 1.14$ \\
\hline LA1313 & $\mathrm{E}$ & Peru & medium & $4.46 \pm 0.73$ & $5.31 \pm 0.59$ & $6.72 \pm 1.46$ \\
\hline LA1315 & $\mathrm{E}$ & Peru & very small & $5.79 \pm 0.23$ & $6.26 \pm 0.32$ & $10.07 \pm 1.89$ \\
\hline LA 1323 & $\mathrm{E}$ & Peru & very small & $4.58 \pm 0.52$ & $5.76 \pm 0.30$ & $8.44 \pm 2.88$ \\
\hline LA 1385 & $\mathrm{E}$ & Peru & small & $5.50 \pm 0.44$ & $5.89 \pm 0.65$ & $8.23 \pm 0.41$ \\
\hline LA 1455 & $\mathrm{E}$ & Mexico & small & $5.04 \pm 0.44$ & $5.79 \pm 0.61$ & $8.11 \pm 1.32$ \\
\hline LA 1456 & $\mathrm{E}$ & Mexico & very small & $5.59 \pm 0.43$ & $6.08 \pm 0.40$ & $8.52 \pm 1.35$ \\
\hline LA 1457 & $\mathrm{E}$ & Mexico & very small & $4.93 \pm 0.35$ & $5.94 \pm 0.37$ & $8.60 \pm 1.25$ \\
\hline LA 1459 & $\mathrm{E}$ & Mexico & small & $5.02 \pm 0.29$ & $4.46 \pm 0.61$ & $7.76 \pm 1.03$ \\
\hline LA721 & $\mathrm{E}$ & Peru & small & $4.49 \pm 0.29$ & $4.58 \pm 0.44$ & $7.21 \pm 0.59$ \\
\hline LA408 & $\mathrm{E}$ & Ecuador & small & $3.53 \pm 0.61$ & $4.00 \pm 0.27$ & $7.58 \pm 0.86$ \\
\hline PI 117897 & $\mathrm{E}$ & Brazil & medium & $3.57 \pm 0.56$ & $3.48 \pm 0.34$ & $5.10 \pm 0.80$ \\
\hline PI 118404 & $\mathrm{E}$ & Venezuela & medium & $3.30 \pm 0.42$ & $4.09 \pm 0.31$ & $8.29 \pm 0.45$ \\
\hline PI 120256 & $\mathrm{E}$ & Turkey & very large & $3.92 \pm 0.30$ & $3.76 \pm 0.40$ & $7.65 \pm 1.70$ \\
\hline PI 126410 & $\mathrm{E}$ & Panama & medium & $4.48 \pm 0.31$ & $5.01 \pm 0.52$ & $7.38 \pm 0.83$ \\
\hline PI 127825 & $\mathrm{E}$ & Bolivia & large & $3.93 \pm 0.33$ & $4.55 \pm 0.18$ & $9.43 \pm 1.37$ \\
\hline PI 128285 & $\mathrm{E}$ & Argentina & large & $3.27 \pm 0.64$ & $3.62 \pm 0.24$ & $6.20 \pm 1.12$ \\
\hline PI 129128 & $\mathrm{E}$ & Panama & large & $3.75 \pm 0.40$ & $3.66 \pm 0.43$ & $10.27 \pm 2.14$ \\
\hline PI 138618 & $\mathrm{E}$ & Iran & very large & $4.42 \pm 0.52$ & $4.06 \pm 0.42$ & $9.39 \pm 3.20$ \\
\hline PI 140051 & $\mathrm{E}$ & Brazil & very large & $3.17 \pm 0.33$ & $3.61 \pm 0.41$ & $7.89 \pm 0.71$ \\
\hline PI 158760 & $\mathrm{E}$ & China & large & $3.76 \pm 0.31$ & $3.37 \pm 0.32$ & $6.95 \pm 1.19$ \\
\hline PI 208837 & $\mathrm{E}$ & Cuba & medium & $3.34 \pm 0.82$ & $3.55 \pm 0.30$ & $8.02 \pm 1.00$ \\
\hline PI 258474 & $\mathrm{E}$ & Ecuador & medium & $3.19 \pm 0.52$ & $3.28 \pm 0.45$ & $5.79 \pm 0.47$ \\
\hline Nagcarlan & $\mathrm{E}$ & Philippines & very large & $3.54 \pm 0.33$ & $3.75 \pm 0.42$ & $7.24 \pm 1.66$ \\
\hline LA 1502 & $\mathrm{E}$ & USA & very large & $3.88 \pm 0.13$ & $3.84 \pm 0.18$ & $8.29 \pm 1.33$ \\
\hline PI 298943 & $\mathrm{E}$ & Poland & large & $4.13 \pm 0.30$ & $3.89 \pm 0.28$ & $1.02 \pm 0.25$ \\
\hline Coldset & $\mathrm{E}$ & USA & large & $2.30 \pm 0.31$ & $2.74 \pm 0.48$ & $5.72 \pm 0.62$ \\
\hline Saladette & $\mathrm{E}$ & USA & large & $4.01 \pm 0.22$ & $3.14 \pm 0.31$ & $5.02 \pm 0.56$ \\
\hline Magnitlo tente & $\mathrm{E}$ & Trinidad & very large & $3.72 \pm 0.36$ & $3.20 \pm 0.47$ & $4.51 \pm 0.69$ \\
\hline PI 406952 & $\mathrm{E}$ & Nicaragua & large & $3.44 \pm 0.65$ & $3.11 \pm 0.40$ & $5.91 \pm 1.02$ \\
\hline Walter & $\mathrm{E}$ & USA & very large & $3.61 \pm 0.37$ & $2.96 \pm 0.41$ & $4.15 \pm 0.47$ \\
\hline UC204A & $\mathrm{E}$ & USA & large & $3.56 \pm 0.45$ & $2.80 \pm 0.28$ & $5.83 \pm 0.95$ \\
\hline CLN2026D & $\mathrm{E}$ & Taiwan & large & $3.30 \pm 0.33$ & $3.37 \pm 0.27$ & $6.89 \pm 0.35$ \\
\hline FMTT 33 & $\mathrm{E}$ & Taiwan & very large & $3.55 \pm 0.37$ & $3.36 \pm 0.34$ & $5.09 \pm 0.66$ \\
\hline NCEBR-6 $(o g c)$ & $\mathrm{E}$ & USA & large & $2.80 \pm 0.48$ & $3.22 \pm 0.56$ & $4.76 \pm 0.61$ \\
\hline $\mathrm{T} 4065(h p, o g c)$ & $\mathrm{E}$ & USA & very large & $2.42 \pm 0.54$ & $2.70 \pm 0.28$ & $10.64 \pm 1.36$ \\
\hline $\mathrm{T} 5019(d g, o g c)$ & $\mathrm{E}$ & USA & very large & $3.40 \pm 0.69$ & $3.91 \pm 0.37$ & $9.30 \pm 1.30$ \\
\hline CLN1314G $(B)$ & $\mathrm{E}$ & Taiwan & very large & $2.86 \pm 0.80$ & $3.23 \pm 0.32$ & $1.18 \pm 0.04$ \\
\hline 97L63 $(B)$ & $\mathrm{E}$ & USDA & large & $1.77 \pm 1.09$ & $2.53 \pm 0.16$ & $0.28 \pm 0.08$ \\
\hline LA2996A $(D e l)$ & $\mathrm{E}$ & USA & very large & $3.76 \pm 0.29$ & $3.12 \pm 0.69$ & $2.41 \pm 0.84$ \\
\hline LA2999 $(g f)$ & $\mathrm{E}$ & USA & large & $3.89 \pm 0.25$ & $2.71 \pm 0.11$ & $3.37 \pm 0.52$ \\
\hline LA2997 $(r)$ & $\mathrm{E}$ & USA & large & $3.99 \pm 0.23$ & $2.77 \pm 0.11$ & $0.04 \pm 0.02$ \\
\hline LA2644 $(s h)$ & $\mathrm{E}$ & USA & very small & $4.69 \pm 0.32$ & $3.84 \pm 0.10$ & $0.05 \pm 0.00$ \\
\hline Waller-Duncan & & & & 1.36 & 1.16 & 2.68 \\
\hline Mean of all entries & & & & $3.97 \pm 0.08$ & $4.11 \pm 0.09$ & $7.21 \pm 0.31$ \\
\hline
\end{tabular}

zhp = high pigment, $\operatorname{og}^{c}=$ crimson, $d g=$ dark green, $B=$ Beta, Del = Delta, $g f=$ green flesh, $r=$ yellow flesh, $s h=$ sherry.

y $=$ Lycopersicon pimpinellifolium and $\mathrm{E}=$ L. esculentum.

xFruit size estimated visually as: very small $<10 \mathrm{~g}$, small 11-30 g, medium 31-50 g, large 51-90 g, very large $>90 \mathrm{~g}$.

wILP = inhibition of lipid peroxidation and $\mathrm{ARP}=$ anti-radical power.

vValues for $\mathrm{a}$ and $\mathrm{b}$ were measured with a chromometer using a red standard surface. Immature green tomatoes have an $\mathrm{a} / \mathrm{b}$ ratio less than zero. The $\mathrm{a} / \mathrm{b}$ ratio 
Vegetable Center, Taiwan, in 2001-02 and 2002-03. Values are means ( \pm SE) averaged over years.

\begin{tabular}{|c|c|c|c|c|}
\hline \multicolumn{3}{|c|}{ Antioxidants } & \multicolumn{2}{|c|}{ Fruit qualities } \\
\hline$\beta$-carotene & Phenolics & $\begin{array}{c}\text { Ascorbic } \\
\text { acid }\end{array}$ & $\begin{array}{l}\text { Color } \\
(\mathrm{a} / \mathrm{b})^{\mathrm{v}}\end{array}$ & $\begin{array}{c}\text { Soluble solids } \\
(\%)\end{array}$ \\
\hline
\end{tabular}

\begin{tabular}{|c|c|c|c|c|}
\hline $0.88 \pm 0.18$ & $156 \pm 22$ & $39.7 \pm 5.6$ & $2.09 \pm 0.19$ & $7.7 \pm 0.15$ \\
\hline $0.89 \pm 0.19$ & $161 \pm 20$ & $36.6 \pm 4.4$ & $2.07 \pm 0.05$ & $8.6 \pm 1.20$ \\
\hline $0.89 \pm 0.09$ & $143 \pm 12$ & $28.5 \pm 3.4$ & $1.99 \pm 0.02$ & $7.1 \pm 0.80$ \\
\hline $0.46 \pm 0.10$ & $77 \pm 2$ & $15.3 \pm 3.6$ & $1.94 \pm 0.07$ & $4.1 \pm 0.20$ \\
\hline $0.32 \pm 0.05$ & $70 \pm 3$ & $13.6 \pm 2.4$ & $1.95 \pm 0.09$ & $3.7 \pm 0.09$ \\
\hline $0.64 \pm 0.05$ & $70 \pm 4$ & $14.6 \pm 1.8$ & $1.36 \pm 0.08$ & $3.8 \pm 0.11$ \\
\hline $0.46 \pm 0.06$ & $70 \pm 3$ & $15.2 \pm 1.2$ & $1.35 \pm 0.10$ & $4.2 \pm 0.18$ \\
\hline $0.75 \pm 0.11$ & $96 \pm 2$ & $19.8 \pm 3.4$ & $1.53 \pm 0.10$ & $4.2 \pm 0.15$ \\
\hline $0.55 \pm 0.09$ & $96 \pm 5$ & $21.8 \pm 3.7$ & $1.62 \pm 0.06$ & $5.3 \pm 0.13$ \\
\hline $0.75 \pm 0.08$ & $105 \pm 8$ & $21.3 \pm 2.3$ & $1.49 \pm 0.10$ & $4.4 \pm 0.27$ \\
\hline $1.16 \pm 0.19$ & $117 \pm 7$ & $22.6 \pm 3.2$ & $1.47 \pm 0.13$ & $4.8 \pm 0.24$ \\
\hline $0.41 \pm 0.03$ & $98 \pm 6$ & $17.5 \pm 1.5$ & $1.68 \pm 0.14$ & $5.1 \pm 0.19$ \\
\hline $0.66 \pm 0.11$ & $143 \pm 11$ & $26.5 \pm 2.5$ & $1.72 \pm 0.16$ & $6.3 \pm 0.35$ \\
\hline $0.83 \pm 0.22$ & $118 \pm 7$ & $24.3 \pm 1.4$ & $1.58 \pm 0.00$ & $5.0 \pm 0.10$ \\
\hline $0.66 \pm 0.05$ & $130 \pm 22$ & $22.5 \pm 3.3$ & $1.63 \pm 0.05$ & $4.7 \pm 0.41$ \\
\hline $1.00 \pm 0.16$ & $123 \pm 22$ & $28.6 \pm 2.4$ & $1.41 \pm 0.10$ & $5.0 \pm 0.41$ \\
\hline $1.15 \pm 0.09$ & $134 \pm 14$ & $26.2 \pm 4.3$ & $0.99 \pm 0.02$ & $4.4 \pm 0.10$ \\
\hline $1.10 \pm 0.08$ & $121 \pm 9$ & $27.9 \pm 5.0$ & $1.49 \pm 0.14$ & $5.9 \pm 0.48$ \\
\hline $0.73 \pm 0.12$ & $101 \pm 8$ & $20.1 \pm 3.5$ & $1.67 \pm 0.12$ & $5.4 \pm 0.50$ \\
\hline $0.68 \pm 0.05$ & $103 \pm 5$ & $22.9 \pm 2.2$ & $1.56 \pm 0.11$ & $4.7 \pm 0.41$ \\
\hline $0.58 \pm 0.05$ & $90 \pm 4$ & $21.8 \pm 3.8$ & $1.52 \pm 0.11$ & $4.6 \pm 0.15$ \\
\hline $0.53 \pm 0.03$ & $79 \pm 8$ & $17.0 \pm 2.3$ & $1.43 \pm 0.14$ & $4.8 \pm 0.17$ \\
\hline $0.54 \pm 0.11$ & $85 \pm 4$ & $16.5 \pm 4.2$ & $1.79 \pm 0.05$ & $5.0 \pm 0.11$ \\
\hline $0.69 \pm 0.08$ & $82 \pm 7$ & $18.3 \pm 3.1$ & $1.75 \pm 0.15$ & $4.5 \pm 0.13$ \\
\hline $0.67 \pm 0.02$ & $103 \pm 5$ & $22.2 \pm 3.0$ & $1.54 \pm 0.09$ & $4.1 \pm 0.20$ \\
\hline $0.62 \pm 0.10$ & $91 \pm 1$ & $19.4 \pm 4.2$ & $1.85 \pm 0.12$ & $4.5 \pm 0.19$ \\
\hline $0.49 \pm 0.13$ & $77 \pm 3$ & $15.3 \pm 2.2$ & $1.89 \pm 0.12$ & $4.4 \pm 0.05$ \\
\hline $0.30 \pm 0.01$ & $92 \pm 7$ & $26.4 \pm 5.1$ & $2.13 \pm 0.13$ & $5.3 \pm 0.31$ \\
\hline $0.60 \pm 0.07$ & $121 \pm 35$ & $20.9 \pm 2.4$ & $1.70 \pm 0.21$ & $5.3 \pm 0.74$ \\
\hline $0.37 \pm 0.05$ & $79 \pm 4$ & $16.4 \pm 3.2$ & $1.90 \pm 0.14$ & $4.4 \pm 0.11$ \\
\hline $0.47 \pm 0.04$ & $71 \pm 8$ & $14.0 \pm 1.5$ & $1.81 \pm 0.09$ & $5.0 \pm 0.13$ \\
\hline $0.48 \pm 0.02$ & $69 \pm 10$ & $15.7 \pm 2.7$ & $1.89 \pm 0.14$ & $4.9 \pm 0.20$ \\
\hline $0.42 \pm 0.04$ & $75 \pm 3$ & $17.3 \pm 3.3$ & $1.80 \pm 0.09$ & $4.2 \pm 0.32$ \\
\hline $0.70 \pm 0.15$ & $88 \pm 5$ & $20.8 \pm 4.1$ & $1.70 \pm 0.17$ & $4.7 \pm 0.26$ \\
\hline $0.48 \pm 0.04$ & $81 \pm 3$ & $16.9 \pm 2.0$ & $1.88 \pm 0.14$ & $4.6 \pm 0.46$ \\
\hline $2.93 \pm 0.33$ & $82 \pm 5$ & $16.3 \pm 2.3$ & $0.63 \pm 0.08$ & $3.8 \pm 0.21$ \\
\hline $0.28 \pm 0.06$ & $65 \pm 2$ & $14.6 \pm 1.9$ & $1.78 \pm 0.13$ & $3.7 \pm 0.09$ \\
\hline $0.47 \pm 0.04$ & $67 \pm 3$ & $15.8 \pm 1.7$ & $1.65 \pm 0.12$ & $4.0 \pm 0.17$ \\
\hline $0.28 \pm 0.05$ & $64 \pm 2$ & $11.8 \pm 2.6$ & $1.77 \pm 0.19$ & $5.1 \pm 0.27$ \\
\hline $0.39 \pm 0.05$ & $96 \pm 20$ & $15.6 \pm 3.1$ & $1.78 \pm 0.16$ & $4.6 \pm 0.14$ \\
\hline $0.36 \pm 0.04$ & $63 \pm 4$ & $12.1 \pm 1.9$ & $1.53 \pm 0.13$ & $4.7 \pm 0.30$ \\
\hline $0.42 \pm 0.05$ & $63 \pm 1$ & $12.3 \pm 2.7$ & $1.72 \pm 0.15$ & $3.7 \pm 0.18$ \\
\hline $0.37 \pm 0.05$ & $74 \pm 1$ & $15.0 \pm 2.5$ & $1.74 \pm 0.06$ & $3.6 \pm 0.14$ \\
\hline $0.44 \pm 0.07$ & $72 \pm 1$ & $14.2 \pm 2.5$ & $1.62 \pm 0.14$ & $4.6 \pm 0.09$ \\
\hline $0.27 \pm 0.07$ & $60 \pm 1$ & $11.8 \pm 1.8$ & $1.73 \pm 0.11$ & $3.8 \pm 0.25$ \\
\hline $0.56 \pm 0.03$ & $63 \pm 4$ & $18.0 \pm 1.7$ & $2.16 \pm 0.17$ & $4.6 \pm 0.06$ \\
\hline $1.15 \pm 0.14$ & $87 \pm 3$ & $17.5 \pm 2.6$ & $1.81 \pm 0.19$ & $4.2 \pm 0.21$ \\
\hline $3.73 \pm 0.28$ & $68 \pm 3$ & $13.4 \pm 2.6$ & $0.73 \pm 0.02$ & $4.2 \pm 0.14$ \\
\hline $3.38 \pm 0.33$ & $56 \pm 4$ & $12.9 \pm 2.8$ & $0.47 \pm 0.03$ & $4.2 \pm 0.09$ \\
\hline $0.20 \pm 0.03$ & $73 \pm 1$ & $11.6 \pm 1.7$ & $0.89 \pm 0.23$ & $5.1 \pm 0.25$ \\
\hline $0.34 \pm 0.05$ & $64 \pm 3$ & $12.0 \pm 0.7$ & $1.34 \pm 0.29$ & $4.6 \pm 0.27$ \\
\hline $0.04 \pm 0.01$ & $64 \pm 0$ & $13.5 \pm 1.1$ & $-0.22 \pm 0.01$ & $4.7 \pm 0.26$ \\
\hline $0.16 \pm 0.01$ & $91 \pm 1$ & $29.9 \pm 2.3$ & $-0.08 \pm 0.01$ & $6.0 \pm 0.20$ \\
\hline 0.29 & 29 & 5.30 .27 & 1.0 & \\
\hline $0.73 \pm 0.05$ & $90 \pm 2$ & $19.1 \pm 0.6$ & $1.55 \pm 0.04$ & $4.8 \pm 0.07$ \\
\hline
\end{tabular}

increases to zero and above as fruit color becomes a dark red.
25 methanol $(\mathrm{v} / \mathrm{v})$ at a flow rate of $1.5 \mathrm{~mL} \cdot \mathrm{min}^{-1}$. Commercial $\beta$-carotene and lycopene were used as standards.

Ascorbic ACID. The determination of total ascorbic acid was on the basis of coupling 2,4dinitrophenylhydrazine (DNPH) with the ketonic groups of dehydroascorbic acid through the oxidation of ascorbic acid by 2,6-dichlorophenolindophenol(DCPIP) to form a yellow-orange color in acidic conditions (Pelletier, 1985). Twenty grams of frozen slurry was blended with 80 $\mathrm{mL}, 5 \%$ meta-phosphoric acid in a homogenizer and centrifuged. After centrifuging, $2 \mathrm{~mL}$ of the supernatant was poured into a $20 \mathrm{~mL}$ test tube containing $0.1 \mathrm{~mL}$ of $0.2 \%$ 2,6-DCPIP sodium salt in water, $2 \mathrm{~mL}$ of $2 \%$ thiourea in $5 \%$ metaphosphoric acid and $1 \mathrm{~mL}$ of 4\% 2,4-DNPH in $9 \mathrm{~N}$ sulfuric acid. The mixtures were kept in a water bath at $37^{\circ} \mathrm{C}$ for $3 \mathrm{~h}$ followed by an ice bath for $10 \mathrm{~min}$. Five milliliters of $85 \%$ sulfuric acid was added and the mixtures were kept at room temperature for $30 \mathrm{~min}$ before reading at OD $520 \mathrm{~nm}$. 2,4-DNPH was added during the ice bath as a blank for a control. Commercial L-(+)-ascorbic acid was used for calibration.

Total Phenolics. Total soluble phenolics were extracted from frozen tomato slurry with methanol and determined using Folin-Ciocalteu reagent (Singleton and Rossi, 1965). The reaction mixtures included $0.2 \mathrm{~mL}$ methanol extract, 3.2 $\mathrm{mL}$ distilled water, $0.2 \mathrm{~mL} 1 \mathrm{~N}$ Folin-Ciocalteu reagent, and $0.4 \mathrm{~mL} 35 \%$ sodium carbonate in water. The absorbance was read at $760 \mathrm{~nm}$ after 30 min incubation at room temperature. Chlorogenic acid was used for quantification.

COLOR AND SOLUBLE SOLIDS CONCENTRATION. Color was measured by a colorimeter (Nippon Denshoku Kogyo Co., Ltd. Osaka, Japan) on three scales represented as a, b, and L. Color values of fresh tomato slurry were calculated as $\mathrm{a} / \mathrm{b}$. Soluble solids concentration was measured with a digital refractometer (PR-101; Atago, Tokyo, Japan).

Statistical analyses. ARP, ILP, lycopene, $\beta$-carotene, ascorbic acid, phenolics, soluble solids concentration, and color were subjected to analysis of variance (ANOVA) for each year and over years with the SAS General Linear Model (GLM) procedure (SAS Institute, Cary, N.C.). For the ANOVA over years, year was regarded as a random effect, entry as a fixed effect, and the year $\times$ entry interaction mean square was used to test the significance of the entry mean square and linear contrasts. Nonindependent, singledegree-of-freedom contrasts were constructed to make comparisons among groups of entries: 1) L. pimpinellifolium $(\mathrm{n}=3)$ vs. L. esculentum entries $(\mathrm{n}=50) ; 2$ ) very small and small redfruited $L$. esculentum $(\mathrm{n}=12)$ vs. medium, large, very large red-fruited L. esculentum (n = $31)$; 3$)$ red fruited $(n=43)$ vs. high $\beta$-carotene $(\mathrm{n}=3)$ entries; and 4) $h p$ and $d g(\mathrm{n}=2)$ entries 
vs. other large and very large, red-fruited $(n=29)$ entries. Entry means were separated by the Waller-Duncan test $(\mathrm{k}=100$, which is equivalent to $P=0.05)$. Linear correlations between characters were calculated with entry means averaged over years. The three L. pimpinellifolium entries LA1582, LA1586, LA1593) and the three Beta gene entries (PI298943, CLN1314G, 97L63) were dropped before calculation of correlations because plotting of means indicated that they were outliers and did not fit the trend of the other entries.

\section{Results}

ANOVA over 2 years (Table 2) revealed highly significant differences among entries for all characters, reflecting the large diversity among entries. Except for ascorbic acid content, trial means for characters were similar between years and year mean squares were nonsignificant. Year $\times$ entry interactions were highly significant for most characters although variance components for entries were at least four times larger than their respective year $\times$ entry variances (data not shown), indicating that variation due to genetic causes was more important than the genotype $\times$ environment interactions.

Antioxidant ACTIVITY. Entry means over years in for ILP ranged from 1.8 to $5.8 \mathrm{TE} \mu \mathrm{mol} \cdot \mathrm{g}^{-1}$ and ARP values over years ranged from 2.5 to $6.8 \mathrm{TE} \mu \mathrm{mol} \cdot \mathrm{g}^{-1}$ (Table 1 ). In general, our ARP values, given in $\mu \mathrm{mol} \cdot \mathrm{g}^{-1}$, were much lower than the equivalent values given in $\mathrm{mmol} \cdot \mathrm{g}^{-1}$ reported by Minoggio et al. (2003). ILP and ARP means in our study for most entries were slightly larger in the 2002 trial than in 2003 (data not shown), suggesting environmental effects on expression of AOA. Mean temperatures in both trials were similar but plants in the 2002 trial were infected with geminivirus in earlier plant stages, resulting in reduced vine cover and more fruit exposure to sunlight. The group mean of the three L. pimpinellifolium accessions (LA1582, LA1586, and LA1593) over years was significantly greater than the mean of the L. esculentum entries for both ILP and ARP (Table 2, contrast 1). LA1582, LA1586, and LA1593 ranked first, second, and third highest, respectively for ARP, and second, eleventh, and seventh, respectively for ILP. Small-fruited L. esculentum entries $(<31 \mathrm{~g})$ produced significantly higher AOA than the medium and large-fruited entries (Table 2, contrast 2). LA1315, LA1385, LA1455, LA1456, and LA1457, all of which are small-fruited and classified as L. esculentum var. cerasiforme (Dun.) A. Gray or South American landraces, demonstrated the highest levels of AOA among L. esculentum entries. All medium, large, and very large-fruited entries showed low or moderate AOA.

LyCOPENE AND $\beta$-CAROTENE CONTENT. For most entries, lycopene was the major carotenoid although the range of values was wide [0.04 to $23.09 \mathrm{mg} / 100 \mathrm{~g}$ (fresh weight basis)]. The three $L$. pimpinellifolium entries formed a distinct group of means that were significantly larger than all the L. esculentum entries (Tables 1 and 2, contrast 1). The L. esculentum means ranged from 0.04 to $10.64 \mathrm{mg} / 100 \mathrm{~g}$. Lycopersicon esculentum entries producing high lycopene content included T5019 and T4065 containing $d g$

Table 2. Mean squares ${ }^{z}$ from analysis of variance and linear contrasts of Lycopersicon entries evaluated for antioxidant activity, antioxidants, and fruit quality components, AVRDC - The World Vegetable Center, Taiwan, in 2002-03.

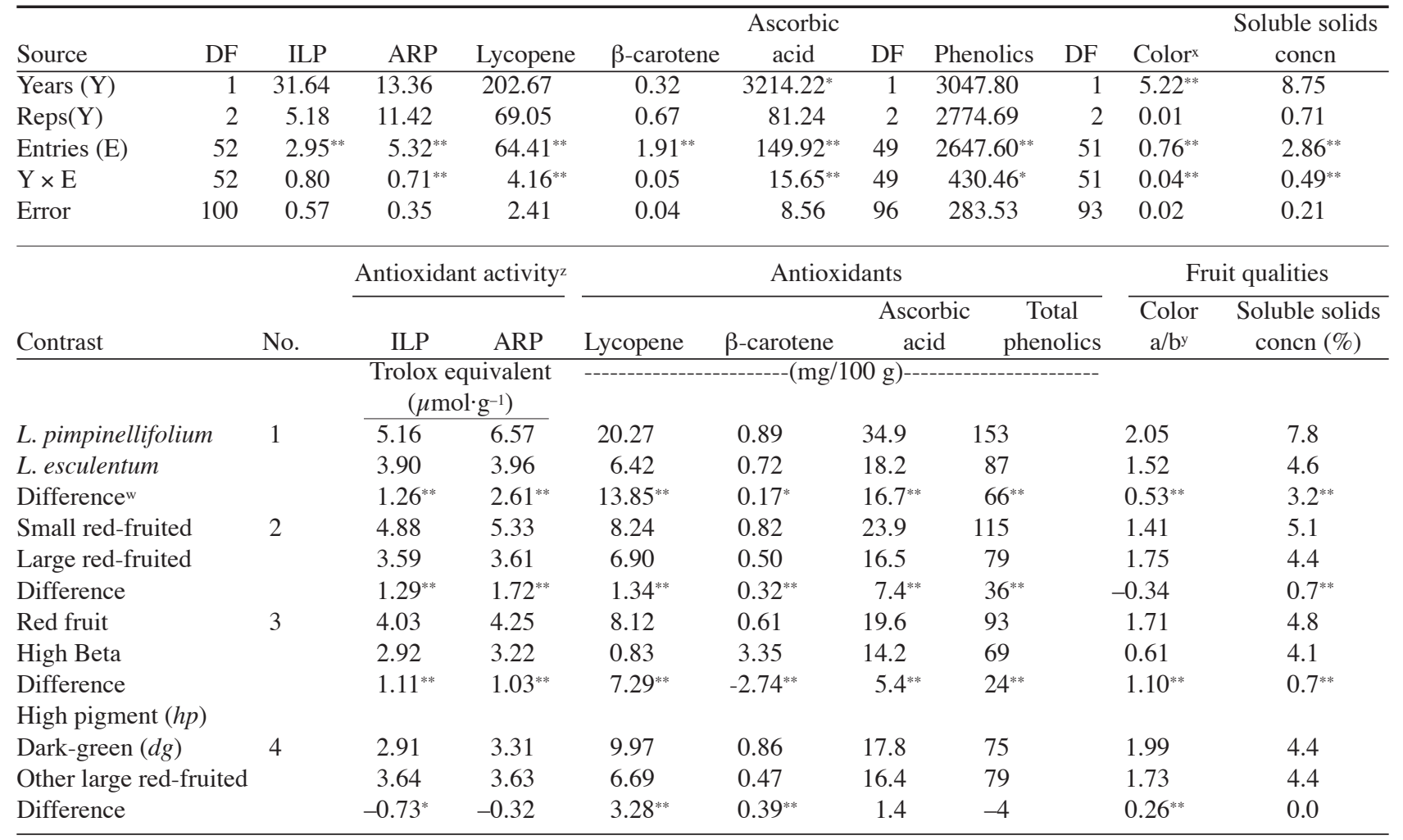

${ }^{\mathrm{z}} \mathrm{ARP}=$ anti-radical power; ILP $=$ inhibition of lipid peroxidation.

vValues for $\mathrm{a}$ and $\mathrm{b}$ were measured with a chromometer using a red standard surface. Immature green tomatoes have an a/b ratio less than zero. The $\mathrm{a} / \mathrm{b}$ ratio increases to zero and above as fruit color becomes a dark red.

*,**Indicates that the difference between group means was significant at $P=0.05$ and $P=0.01$, respectively, according to a single-degree-of-freedom linear contrast.

${ }^{*},{ }^{* *}$ Mean square significant at $P=0.05$ and 0.01 , respectively. 
Table 3. Linear correlations ${ }^{z}$ determined between two measures of antioxidant activity, four antioxidants, and three fruit quality components of Lycopersicon entries at AVRDC - The World Vegetable Center, in 2002-03.

\begin{tabular}{|c|c|c|c|c|c|c|c|c|}
\hline & ILPy & Lycopene & $\beta$-carotene & Ascorbic acid & Phenolics & Color & Soluble solids & Fruit size \\
\hline$\overline{\mathrm{ARP}}$ & $0.82^{* *}$ & $0.49^{* *}$ & $0.70^{* *}$ & $0.69^{* *}$ & $0.90^{* *}$ & 0.01 & $0.43^{\text {** }}$ & $-0.74^{* *}$ \\
\hline ILP & & 0.17 & $0.53^{* *}$ & $0.65^{\text {** }}$ & $0.83^{* *}$ & -0.22 & $0.54^{* *}$ & $-0.71^{* *}$ \\
\hline Lycopene & & & $0.53^{* *}$ & 0.24 & $0.44^{* *}$ & $0.75^{* *}$ & 0.13 & -0.16 \\
\hline$\beta$-carotene & & & & $0.59^{\text {** }}$ & $0.70^{\text {*** }}$ & 0.18 & 0.18 & $-0.49^{* *}$ \\
\hline Ascorbic acid & & & & & $0.81^{* *}$ & -0.20 & $0.57^{* *}$ & $-0.74^{* *}$ \\
\hline Phenolics & & & & & & -0.03 & $0.54^{* *}$ & $-0.72^{* *}$ \\
\hline Color & & & & & & & -0.19 & 0.23 \\
\hline Soluble solids & & & & & & & & $-0.46^{* *}$ \\
\hline
\end{tabular}

${ }^{\mathrm{z}}$ Correlations were calculated using entry means averaged over 2 years. The three L. pimpinellifolium and the three entries with the Beta genes were dropped before calculation of correlations.

yARP $=$ anti-radical power; ILP $=$ inhibition of lipid peroxidation.

${ }^{*}, *$ Significant at $P=0.05$ and 0.01 , respectively; $\mathrm{n}=47$.

$+o g^{\mathrm{c}}$ and $h p+o g^{\mathrm{c}}$ genes, respectively, as well as LA1315 and PI129128.

As expected, entries CLN1314G and 97L63 containing the Beta gene showed low lycopene density and the highest $\beta$-carotene levels (3.73 and $3.38 \mathrm{mg} / 100 \mathrm{~g}$, respectively) compared to an average $\beta$-carotene content of $0.61 \mathrm{mg} / 100 \mathrm{~g}$ for the red-fruited entries. A third entry, PI298943, probably has Beta or a gene similar to Beta since it produced orange fruit with a $\beta$-carotene content of $2.93 \mathrm{mg} / 100 \mathrm{~g}$. The Beta gene in cultivated tomato was derived from interspecific crosses with wild Lycopersicon species (Stommel and Haynes, 1994). Beta in CLN1314G and 97L63 originated from L. hirsutum Humb. and Bonpl. (Ali and Tsou, 2000) and L. cheesmanii Riley (Stommel and Haynes, 1994), respectively. Passport information for PI298943 (USDA, ARS, 2004) lists this accession as a derivative from an interspecific cross of L. esculentum $\times$ L. minutum; the "L. minutum complex" includes L. parviflorum Rick, Kesicki, Fobes \& Holle and $L$. chmielewskii Rick, Kesicki, Fobes \& Holle (Rick et al., 1976), and it is likely the Beta gene in this accession was introduced from L. minutum. Among L. esculentum entries, T5019 $(d g+o g c)$ produced two times more $\beta$-carotene than the normal red-fruited tomato. This observation is in agreement with Wann et al. (1985) who detected elevated levels of lycopene, $\beta$-carotene and vitamin $\mathrm{C}$ in lines containing $h p+o g^{\mathrm{c}}$ and $d g+o g^{\mathrm{c}}$. However, T4065 $(h p$ $+o g c$ ) showed normal levels of $\beta$-carotene in this study.

Phenolics AND ASCORbIC ACID. Entry means over years for phenolics content ranged from 56 to $156 \mathrm{mg} / 100 \mathrm{~g}$ (fresh weight basis) with an overall mean of $90 \mathrm{mg} / 100 \mathrm{~g}$. In general, our total phenolics data were about five times higher than those reported by Minoggio et al. (2003). Entry means over years for ascorbic acid content ranged from 12 to $40 \mathrm{mg} / 100 \mathrm{~g}$. For both characters, average content of the L. pimpinellifolium entries was almost twice as large as the mean of all L. esculentum entries (Table 2, contrast 1). Almost all L. esculentum entries of relatively high phenolics content were small-fruited and means of almost all medium or large-fruited entries fell below $90 \mathrm{mg} / 100 \mathrm{~g}$. However, several large-fruited entries, including PI406952 and PI138618, produced relatively high levels of phenolics; however, values for PI138618 were inconsistent between years with a mean of $164 \mathrm{mg} / 100 \mathrm{~g}$ in 2002 but only $78 \mathrm{mg} / 100 \mathrm{~g}$ in 2003 . Like phenolics content, entries producing the highest ascorbic acid content included the L. pimpinellifolium and small-fruited L. esculentum such as LA1455 and LA2644. We did not detect high phenolics content in T4065, homozygous for the $h p$ allele that has been associated with higher content of quercetin, a flavonoid, in tomato fruit (Yen et al., 1997). Wann et al. (1985) noted elevated vitamin C content in lines containing $h p+o g^{\mathrm{c}}$ and $d g+o g^{\mathrm{c}}$ but in our study T4065 $\left(h p+o g^{c}\right)$ and T5019 $\left(d g+o g^{c}\right)$ contained only slightly higher ascorbic acid densities compared to other large-fruited entries.

COLOR AND SOLUBLE SOLIDS CONCENTRATION. Group mean color values of the L. pimpinellifolium exceeded the group mean of the L. esculentum entries and was due to greater lycopene densities of the L. pimpinellifolium entries (Table 2, contrast 1). Compared to other large-fruited entries, T5019 and T4065 showed significantly higher color values (Table 2, contrast 4). Like color content, soluble solids concentration of the L. pimpinellifolium entries were significantly higher than the L. esculentum and small fruit as a group produced higher soluble solids concentration than the large-fruited entries.

Correlations. We found a large positive correlation between ARP and ILP (Table 3), indicating a strong linear association between the two measures of AOA. ARP and ILP were both highly correlated with total phenolics in particular but $\beta$-carotene and ascorbic acid were also significantly correlated with both AOA assays. Lycopene showed a significant positive correlation with ARP but not ILP. Except for lycopene, correlations between AO and fruit size were negative and highly significant.

\section{Discussion}

Results of our study have implications for improvement of tomato for AOA. Genetic improvement of tomato for AOA and specific $\mathrm{AO}$ is possible given the large genetic variation present among tomato entries. However, entries outstanding for AOA and most $\mathrm{AO}$ in this study were exclusively L. pimpinellifolium (LA1582, LA1586, and LA 1593) or small-fruited L. esculentum such as LA1315, LA1385, and LA1455, and the medium or largefruited entries in this study showed average or below average AOA. Although information is lacking on the improvement status of many entries, particularly those prefixed with PI (USDA-ARS, 2004) it is almost certain that the large-fruited entries were developed in plant breeding programs while L pimpinellifolium and small-fruited L. esculentum are nonbred germplasm. Further work is required to determine if lower AOA of the larger, and presumably bred lines, is a consequence of increased fruit size or the result of selection against certain AO, especially phenolics, in the course of domestication/plant breeding.

Over half a century ago Lincoln et al. (1943) noted the high contents of vitamin $C$, lycopene, $\beta$-carotene and soluble solids in L. pimpinellifolium. However, these traits are polygenic and introgression of genes improving these traits into elite tomato cultivars by conventional breeding has been difficult. Molecular 
markers have opened up new avenues to exploit genetic resources for crop improvement, particularly in identification of new genes improving quantitative characters (Tanksley and Nelson, 1996). Markers have been applied to identify and map L. pimpinellifolium alleles conditioning deeper red color (Bernacchi et al., 1998; Tanksley et al., 1996) or increased lycopene content (Chen et al., 1999). Marker-based methods such as advanced backcross QTL should permit faster and efficient transfer of beneficial alleles from wild tomato into elite tomato genetic backgrounds (Tanksley and Nelson, 1996).

ILP and ARP, the two methods used to assess AOA in this study, could be adopted by plant breeders to improve AOA in tomato. ARP in particular was positively correlated with all four $\mathrm{AO}$, and use of this AOA assay would be cheaper and easier than measuring all individual AO. Our results suggest that ILP and ARP measure similar properties since there was a large positive correlation between the two assays $(r=0.82 * *)$ and the magnitude and range of ILP and ARP values were similar. This was not the case for Capsicum annuum L. (AVRDC-The World Vegetable Center, unpublished data). Consequently there is no need to evaluate entries with both assays in a breeding program. Of the two methods we recommend ARP because the time required by laboratory technicians to complete this assay was $\approx 50 \%$ less than ILP at about the same material cost. Expression of ILP and ARP were influenced by the environment, evidenced by the significant entry $\times$ year mean squares. In general, ranking of entries for ILP and ARP were consistent between years, particularly for those entries with the highest means; of the top ten entries for ILP in 2002, six were among the top ten in 2003; for ARP, eight of the top 10 entries in 2002 ranked in the top 10 in 2003. Consistent relative performance of entries allows breeders to more efficiently use testing resources, discarding poor entries and focusing future testing on the better entries (Schutz and Bernard, 1967).

Among AO, total phenolics demonstrated the largest positive correlations with both ARP and ILP. The importance of phenolic phytochemicals in AOA of fruit and vegetables has been documented (Cao et al., 1996; Velioglu et al., 1998; Vinson et al., 1998; Weisburger, 1999) and our results and those of Minoggio et al. (2003) indicate a major role of phenolics for AOA in tomato fruit. Tomato breeders have given little attention to the phenolics content of tomato fruit but our study suggests that genetic improvement to increase total phenolics content is a worthy objective. All AO in this study, including total phenolics, were negatively correlated with fruit size, suggesting that development of cultivars of commercial fruit size with high phenolics content may be difficult. Furthermore, phenolic compounds in tomato fruit are concentrated in the skin (Bovy et al., 2002; Davies and Hobson, 1981) and surface area : volume ratio decreases with increasing fruit size (Connor et al., 2002). Further investigations of the location of phenolics in more tomato entries are warranted, especially in L. pimpinellifolium and L. esculentum var. cerasiforme. Although demand for large fruit may impose limits on phenolics concentration, we did find medium and large-fruited entries such as PI138618 and PI406952 with relatively high phenolic values that may be useful in genetic improvement. The existence of mutant genes elevating phenolics content in tomato is also possible and the Aubergine $(\mathrm{Abg})$ gene that produces purpling of the fruit epidermis content is a potential candidate (TGRC, 2004). Ultimately, the transgenic approach developed by Bovy et al. (2002) to induce flavonoid production in tomato fruit may be the best means to obtain high phenolics content in a large-fruited tomato.
Greater realization of the importance of phytochemicals in promotion of human health has prompted the broadening of dietary recommendations to include greater daily intake of a wider range of vegetables and fruit (Peters et al., 2003; Weisburger, 1999). This will be difficult in the tropics and subtropics where vegetable supplies are both highly seasonal and daily per capita availability fall drastically below the $200 \mathrm{~g}$ recommended by AVRDC - The World Vegetable Center (Ali and Tsou, 1997). Increased phytochemical intake in the tropics requires multiple tactics, including production of tropically adapted cultivars with higher densities of micronutrients and AO; overcoming seasonality of vegetable production so affordable vegetables are available to poor consumers throughout the year; diversification of diets to include antioxidant-rich vegetable species such as sweet potato vine [Ipomoea batatas (L.) Lam], Cassia tora L., or chinese cedrela leaves [Toona sinensis (Juss.) M. Roem.] (unpublished data, AVRDC - The World Vegetable Center); and better methods of vegetable preparation and processing in order to boost micronutrient bioavailability. AVRDC-The World Vegetable Center will continue to pursue a leadership role in each of these areas in order to fulfill its mission to "improve nutrition and reduce poverty in the tropics" (AVRDC, 2002)

\section{Literature Cited}

Abushita, A.A., E.A. Hebshi, H.G. Daood, and P.A. Biacs. 1997. Determination of antioxidant vitamins in tomatoes. Food Chem. 60:207-212.

Ali, M. and S.C.S. Tsou. 1997. Combating micronutrient deficiencies through vegetables-A neglected food frontier in Asia. Food Policy $22: 17-38$

Ali, M. and S. Tsou. 2000. The integrated research approach of the Asian Vegetable Research and Development Center (AVRDC) to enhance micronutrient availability. Food Nutr. Bul. 21:472-481.

Arnao, M.B.,A. Cano, and M.Acosta. 2001. The hydrophilic and lipophilic contribution to total antioxidant activity. Food Chem. 73:239-244.

Asian Vegetable Research and Development Center. 2002. Empowering small-scale farmers for knowledge-based agriculture. AVRDC Strategy 2010. AVRDC, Shanhua, Taiwan.

Bernacchi, D., T. Beck-Bunn, D. Emmatty, Y. Eshed, S. Inai, J. Lopez, V. Petiard, H. Sayama, J. Uhlig, D. Zamir, and S. Tanksley. 1998. Advanced backcross QTL analysis of tomato. II. Evaluation of near-isogenic lines carrying single-donor introgressions for desirable wild QTL-alleles derived from Lycopersicon hirsutum and L. pimpinellifolium. Theor. Appl. Genet. 97:170-180.

Bovy, A., R. de Vos, M. Kemper, E. Schijlen, M.A. Pertejo, S. Muir, G. Collins, S. Robinson, M. Verhoeyen, S. Hughes, C. Santos-Buelga, and A. van Tunen. 2002. High-flavonol tomatoes resulting from the heterologous expression of the maize transcription factor genes LC and C1. Plant Cell 14:2509-2526.

Bramley, P.M. 2000. Is lycopene beneficial to human health? Photochemistry 54:233-236.

Cao, G., E. Sofic and R.L. Prior. 1996. Antioxidant capacity of tea and common vegetables. J. Agr. Food Chem. 44:3426-3431.

Chen, F.Q., M.R. Foolad, J. Hyman, D.A. St. Clair, and R.B. Beelaman. 1999. Mapping of QTLs for lycopene and other fruit traits in a Lycopersicon esculentum $\mathrm{x}$ L. pimpinellifolium cross and comparison of QTLs across tomato species. Mol. Breeding 5:283-299.

Clevidence, B.A., F. Khachik, E.D. Brown, P.P. Nair, E.R. Wiley, R.L. Prior, G. Cao, D.W. Morel, W. Stone, M. Gross, and T.R. Kramer. 1997. Human consumption of carotenoid-rich vegetables, p. 53-63. In: O.I. Arouma and S.L. Cuppett (eds.). Antioxidant methodology in vivo and vitro concepts. AOCS Press, Champaign, Ill.

Clinton, S.K. 1998. Lycopene: Chemistry, biology, and implications for human health and disease. Nutr. Rev. 56:35-51.

Connor, A.M., J.J. Luby, C.B.S. Tong, C.E. Finn, and J.F. Hancock. 
2002. Genotypic and environmental variation in antioxidant activity, total phenolics content, and anthocyanin content among blueberry cultivars. J. Amer. Soc. Hort. Sci. 127:89-97.

Davies, J.N. and G.E. Hobson. 1981. The constituents of tomato fruit-The influence of environment, nutrition, and genotype. CRC Critical Rev. in Food Sci. and Nutr. 15:205-280.

Food and Agriculture Organization of the United Nations. 2004. Agricultural data FAOSTAT. 1 Feb. 2004. <http://faostat.fao.org/faostat/ collections?version=ext \&hasbulk $=0 \&$ subset=agriculture $>$

Frankel, E.N. and A.S. Meyer. 2000. The problems of using one-dimensional methods to evaluate multifunctional food and biological antioxidants. J. Sci. Food Agr. 80:1925-1941.

Guo, C., G. Cao, E. Sofic and R.L. Prior. 1997. High-performance liquid chromatography coupled with coulometric array detection of electroactive components in fruits and vegetables: Relationship to oxygen radical absorbance capacity. J. Agr. Food Chem. 45:1787-1796.

Halliwell, B., R. Aeschbach, J. Löliger, and O.I. Arouma. 1995. The characterization of antioxidants. Food Chem. Toxicol. 33:601-617.

Heber, D. and Q-Y. Lu. 2002. Overview of mechanisms of action of lycopene. Expt. Biol. Med. 227:920-923.

Herrmann, K. 1976. Flavonols and flavones in food plants: A review. J. Food Tech. 11:433-448.

Kochian, L.V. and D.F. Garvin. 1999. Agricultural approaches to improving phytonutrient content in plants: An overview. Nutr. Rev. 57: S13-S18.

Lavelli, V., C. Peri, and A. Rizzolo. 2000. Antioxidant activity of tomato products as studied by model reactions using xanthine oxidase, myeloperoxidase, and copper-induced lipid peroxidation. J. Agr. Food Chem. 48:1442-1448.

Lavelli, V., E. Pagliarini, G. Giovanelli, C. Peri, and B. Zanoni. 2001. The antioxidant activity of tomato. I. Evaluation of fresh and processed products by chemical-physical indexes and biochemical model systems through principal component analysis. Acta Hort. 542:205-210.

Leonardi, C., P.Ambrosino, F. Esposito, and V. Fogliano. 2000. Antioxidative activity and carotenoid and tomatine contents in different typologies of fresh consumption tomatoes. J. Agr. Food Chem. 48:4723-4727.

Lincoln, R.E., F.P.Zscheile, J.W. Porter, G.W. Kohler, and R.M. Caldwell. 1943. Provitamin A and vitamin C in the genus Lycopersicon. Bot. Gaz. 105:113-115.

Minoggio M., L. Bramati, P. Simonetti, C. Gardana, L. Iemoli, E. Santangelo, P.L. Mauri, P. Spigno, G.P. Soressi, and P.G. Pietta. 2003. Polyphenol pattern and antioxidant activity of different tomato lines and cultivars. Ann. Nutr. Metabolism 47:64-69.

Omaye S.T. and P. Zhang. 1998. Phytochemical interactions: B-carotene, tocopherol and ascorbic acid, p. 53-75. In: W.R. Bidlack, S.T. Omaye, M.S. Meskin, and D. Jahner (eds.). Phytochemicals - Anew paradigm. Technomic Publishing Co., Lancaster, Pa.

Pelletier O. 1985. Vitamin C(L-ascorbic and dehydro-L-ascorbic acids), p 303-347. In: J. Augustin, B.P. Klein, D.A. Becker, and P.B. Venugopal (eds.). Methods of vitamin assay. $4^{\text {th }}$ ed. Wiley, New York.

Peters, C.J., G.W. Fick, and J.L. Wilkins. 2003. Cultivating better nutrition: Can the food pyramid help translate dietary recommendations into agricultural goals? Agron. J. 95:1424-1430.

Rao, A.V. and S. Agarwal. 1998. Bioavailability and in vivo antioxidant properties of lycopene from tomato products and their possible role in the prevention of cancer. Nutr. Cancer 31:199-203.
Rick, C.M., E. Kesicki, J.F. Fobes, and M. Holle. 1976. Genetic and biosystematic studies on two new sibling species of Lycopersicon from interandean Perú. Theor. Appl. Genet. 47:55-68.

Schutz, W.M. and R.L. Bernard. 1967. Genotype $\times$ environment interactions in the regional testing of soybean strains. Crop Sci. 7:125-130.

Singleton, V.L and J.A. Rossi. 1965. Colorimetry of total phenolics with phosphomolybdic-phosphotungstic acid reagents. Amer. J. Enol. Viticult. 16:144-158

Stahl, W., A. Junghans, B. de Boer, E.S. Driomina, K. Briviba, and H. Sies. 1998. Carotenoid mixtures protect multilamellar liposomes against oxidative damage: Synergistic effects of lycopene and lutein. FEBS Lett. 427:305-308.

Stevens, M.A. 1986. Inheritance of tomato fruit quality components, p. 273-311. In: J. Janick (ed.). Plant breeding rev. vol. 4. AVI, Westport, Conn.

Stevens, M.A. and C.M. Rick. 1986. Genetics and breeding, p. 35-109. In: J.G. Atherton and J. Rudich (eds.). The tomato crop. Univ. Press, Cambridge, U.K.

Stommel, J.R. and K.G. Haynes. 1994. Inheritance of beta carotene content in the wild tomato species Lycopersicon cheesmanii. J. Hered. 85:401-404.

Tanksley, S.D. and J.C. Nelson. 1996. Advanced backcross QTL analysis: A method for the simultaneous discovery and transfer of valuable QTLs from unadapted germplasm into elite breeding lines. Theor. Appl. Genet. 92:191-203.

Tanksley, S.D., S. Grandillo, T.M. Fulton, D. Zamir, Y. Eshed, V. Petiard, J. Lopez, T. Beck-Bunn. 1996. Advanced backcross QTL analysis in a cross between an elite processing line of tomato and its wild relative L. pimpinellifolium. Theor. Appl. Genet. 92:213-224.

Tomato Genetics Resource Center. 2004. C.M. Rick tomato genetics resource center. Univ. California-Davis. 6 Apr. 2004. <http://tgrc. ucdavis.edu/index.cfm>

Tomes, M.L. and F.W. Quackenbush. 1958. 'Caro-red', a new pro-vitamin A rich tomato. Econ. Bot. 12:256-260.

Tucker, G. 2003. Nutritional enhancement of plants. Current Opinion in Biotechnology 14:221-225.

USDA-ARS, National Genetic Resources Program. 2004. Germplasm resources information network (GRIN). (online database) Natl. Germplasm Resources Lab., Beltsville, Md. 6 Apr. 2004. <http://www. ars-grin.gov/cgi-bin/npgs/htmL/tax_site_acc.pl?NE9\%20Lycopersic on\%20esculentum>

Velioglu, Y.S., G. Mazza, L. Gao, and B.D. Oomah. 1998. Antioxidant activity and total phenolics in selected fruits, vegetables, and grain products. J. Agr. Food Chem. 46:4113-4117.

Vinson, J.A., Y.Hao, X. Su and L.Zubik. 1998. Phenol antioxidant quantity and quality in foods: vegetables. J. Agr. Food Chem. 46:3630-3634.

Wann, E.V., E.L. Jourdain, R. Pressey, and B.G. Lyon. 1985. Effect of mutant genotypes $h p o g^{\mathrm{c}}$ and $d g o^{\mathrm{c}}$ on tomato fruit quality. J. Amer. Soc. Hort. Sci. 110:212-215.

Weisburger, J.H. 1999. Mechanisms of action of antioxidants as exemplified in vegetables, tomatoes and tea. Food Chem. Toxicol. 37:943-948.

Yen, H.C., B.A. Shelton, L.R. Howard, S. Lee, J. Vrebalov, and J.J. Giovannoni. 1997. The tomato high-pigment (hp) locus maps to chromosome 2 and influences plastome copy number and fruit quality. Theor. Appl. Genet. 95:1069-1079. 